\title{
The scission point configuration of fissioning nuclei
}

\author{
Fedir Ivanyuk ${ }^{1,2, a}$ \\ ${ }^{1}$ Institute for Nuclear Research, Kiev, Ukraine \\ ${ }^{2}$ Research Laboratory for Nuclear Reactors, Tokyo Institute of Technology, Tokyo, Japan
}

\begin{abstract}
We define the optimal shape which fissioning nuclei attain just before the scission and calculate the deformation energy as function of the mass asymmetry at the scission point. The calculated deformation energy is used in quasi-static approximation for the estimation of mass distribution, total kinetic and excitation energy of fission fragments, and the total number of prompt neutrons. The calculated results reproduce rather well the experimental data on the position of the peaks in the mass distribution of fission fragments, the total kinetic and excitation energy of fission fragments. The calculated value of neutron multiplicity is somewhat larger than experimental results. The saw-tooth structure of neutron multiplicity is qualitatively reproduced.
\end{abstract}

\section{Introduction}

In the theory of nuclear fission the quasistatic quantities like the potential energy surface, the ground state energy and deformation, the fission barrier height are commonly calculated within the macroscopic-microscopic method [1,2]. In this method the total energy of the fissioning nucleus consists of two parts, macroscopic and microscopic. Both parts are calculated at a fixed shape of the nuclear surface. In the past a lot of parameterizations of the shape of nuclear surface were proposed and used. A good choice of the shape parameterization is often a key to the success of the theory. Usually, one relies on the physical intuition for the choice of the shape parameterization.

A method to define the shape of the nuclear surface which does not rely on any shape parameterization was proposed by V. Strutinsky in Ref. [3]. In this approach the shape of an axial, left-right symmetric nucleus was defined by the minimization of the liquid-drop energy under the constraints that fix the elongation of the drop and keep the volume constant.

Recently the method was further developed by incorporating the axial and left-right asymmetry, and account of the influence of shell effects on the shape of nuclear surface, see [4] and references therein.

The important result of the Strutinsky procedure [3] is the possibility to definite in a formal way the scission point as the maximal elongation beyond which the solution of the minimization procedure in form of a compact shape does not exist.

Having at one's disposal the shape and the deformation energy at the scission point and assuming the statistical distribution for the population of points in space of deformation parameters we have evaluated the measurables of the fission experiments: the mass distribution, total kinetic and excitation energy of fission fragments, the multiplicity of prompt neutrons.

\footnotetext{
ae-mail: ivanyuk@kinr.kiev.ua
} 


\section{The optimal shapes of fissioning nuclei}

The shape of an axially symmetric nucleus can be defined by rotation of some profile function $\rho(z)$ around the $z$-axis. It was suggested in [3] to define $\rho(z)$ looking for the minimum of the liquid-drop energy $E_{\mathrm{LD}}=E_{\text {surf }}+E_{\text {Coul }}$ under the constraint that the volume $V$ and the elongation $R_{12}$ are fixed,

$$
\frac{\delta}{\delta \rho}\left(E_{\mathrm{LD}}-\lambda_{1} V-\lambda_{2} R_{12}\right)=0, \quad \text { with } \quad R_{12}=\frac{2 \pi}{V} \int_{z_{1}}^{z_{2}} \rho^{2}(z)|z| d z, \quad V=\pi \int_{z_{1}}^{z_{2}} \rho^{2}(z) d z
$$

The elongation parameter $R_{12}$ was chosen in [3] as the distance between the centers of mass of the left and right parts of the nucleus. In (1) $\lambda_{1}$ and $\lambda_{2}$ are the corresponding Lagrange multipliers.

The minimization of $E_{\mathrm{LD}}-\lambda_{1} V-\lambda_{2} R_{12}$ with respect to the profile function $\rho(z)$ leads to an integrodifferential equation for $\rho(z)$

$$
\rho(z) \frac{\partial^{2} \rho}{\partial z^{2}}=1+\left(\frac{\partial \rho}{\partial z}\right)^{2}-\rho(z)\left[\lambda_{1}+\lambda_{2}|z|-10 x_{\mathrm{LD}} \Phi(z, \rho)\right]\left[1+\left(\frac{\partial \rho}{\partial z}\right)^{2}\right]^{\frac{3}{2}} .
$$

Here $\Phi(z, \rho(z))$ is the Coulomb potential at the nuclear surface and $x_{\mathrm{LD}}$ is the fissility parameter of the liquid drop [5],

$$
x_{\mathrm{LD}} \equiv \frac{E_{\mathrm{Coul}}^{(0)}}{2 E_{\mathrm{surf}}^{(0)}}=\frac{3}{10} \frac{Z^{2} e^{2}}{4 \pi R_{0}^{3} \sigma} \approx \frac{Z^{2}}{49 A},
$$

where $\sigma$ is the surface tension coefficient.
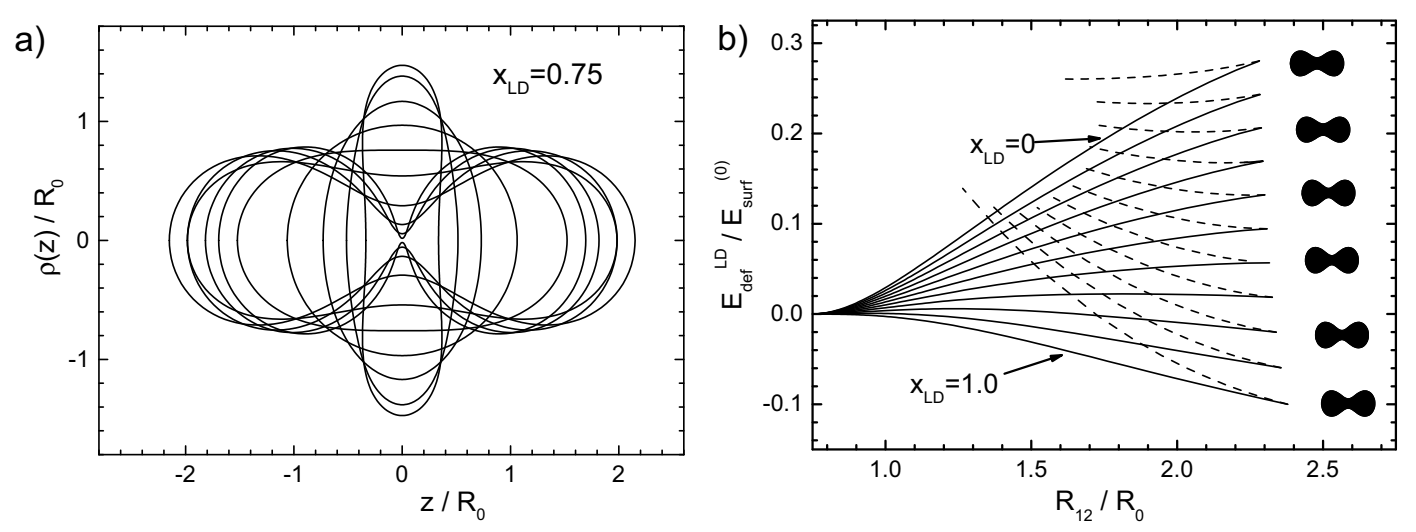

Figure 1. (a) The solutions of Eq. (2) corresponding to different values of Lagrange multiplier $\lambda_{2}$ which fixes the elongation $R_{12}$; (b) Liquid-drop deformation energy (4) as a function of the parameter $R_{12}$ for different values of fissility parameter $x_{\mathrm{LD}}$.

By solving Eq. (2) one obtains the profile function $\rho(z)$ for given $x_{L D}$ and $\lambda_{2}\left(\lambda_{1}\right.$ is fixed by the volume conservation condition). Varying $\lambda_{2}$ one gets a full variety of shapes from the disk, even with central depression, to the two touching spheres, see Fig. 1a. shape)

The liquid drop deformation energy $E_{\mathrm{def}}^{L D}=E_{\mathrm{LD}}-E_{\mathrm{LD}}^{(0)}$ (in units of the surface energy for a spherical

$$
E_{\text {def }}^{L D} / E_{\text {surf }}^{(0)}=B_{\text {surf }}-1+2 x_{\mathrm{LD}}\left(B_{\text {Coul }}-1\right),
$$


calculated for the shapes shown in Fig.1a, is presented in Fig.1b. In (4) $B_{\text {Coul }} \equiv E_{\text {Coul }} / E_{\text {Coul }}^{(0)}, B_{\text {surf }} \equiv$ $E_{\text {surf }} / E_{\text {surf }}^{(0)}$, where an index ${ }^{(0)}$ refers to the spherical shape.

From Fig.1b one can see that the elongation $R_{12}$ of the shapes shown in these figures is limited by some maximal value $R_{12}^{(\text {crit })}$. Above this deformation mono-nuclear shapes do not exist. This critical deformation was interpreted in [3] as the scission point. Note that, at scission the neck radius is still rather large: at the critical deformation the neck radius is approximately equal to $(0.25-0.30) R_{0}$ for a fissility parameter in the range $0.4 \leq x_{L D} \leq 0.9$

Another peculiarity of Fig. 1b is the upper branch of the deformation energy at large deformation. Along this branch the neck of the drop becomes smaller and smaller until the shape turns into two touching spheres. Both branches are solutions of Eq.(2). It turns out, that the upper branch of $E_{\text {def }}^{L D}$ corresponds not to the minimum but to the maximum of the liquid drop energy. Thus, it represents the ridge of the potential energy surface between the fission and fusion valleys.

\section{The mass-asymmetric shapes}

For the description of fission process the account of mass asymmetry is essential. In Fig. 2 we show the deformation energy of ${ }^{236} \mathrm{U}$ calculated within the two-center shell model. The shape in the two-center shell model is fixed by five deformation parameters. This parametrization allows for the description of both compact and separated shapes. The deformation energy in Fig. 2 was minimized with respect all five deformation parameters keeping fixed the mass asymmetry and the distance $R_{12}$ between the centers of mass of left and right parts of nucleus. One can see, that at some value $R_{12}^{(\text {crit })}$ the potential energy drops suddenly to the potential energy of separated fragments, which is much smaller than the potential energy of compact shape.

a)

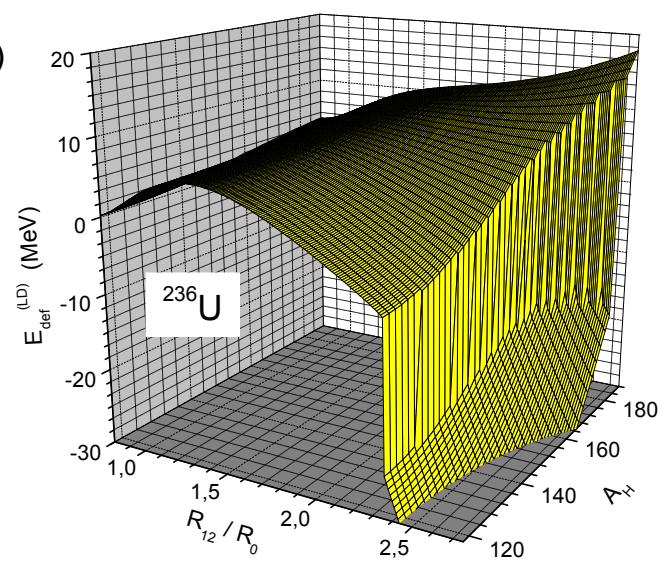

b)

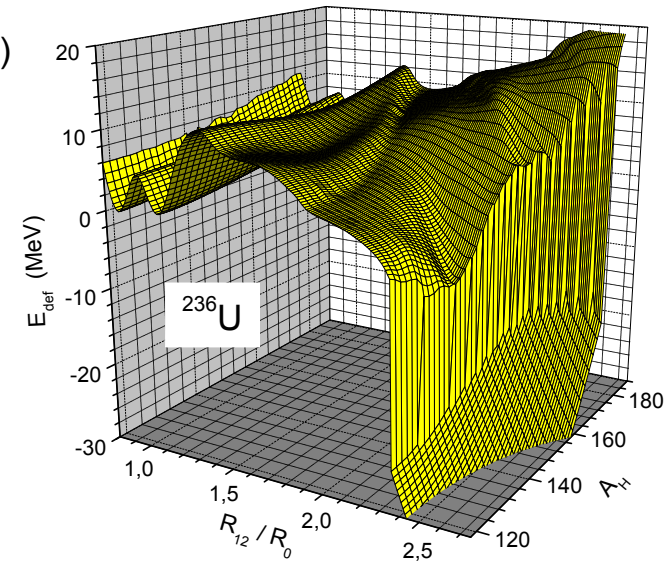

Figure 2. (color online) (a) The liquid-drop deformation energy (4) of ${ }^{236} \mathrm{U}$ as function of the elongation $R_{12}$ and the mass number $A_{H}$ of heavy fragment calculated within the two-center shell model; (b) The total (liquid-drop plus shell correction) deformation energy of ${ }^{236} \mathrm{U}$.

In order to give the model-independent definition of $R_{12}^{(c r i t)}$ one has to generalize the optimal shape approach of [3] to the case of mass-asymmetric shapes. For this aim one has to include into Eq.(1) one more constraint that fix the mass asymmetry $\delta$ of the drop. The mass asymmetry $\delta$ is commonly defined by the difference of masses $M_{L}$ and $M_{R}$ to the left and right of some point $z^{*}$. In case that the 
drop has a neck, $z^{*}$ coincides with the position of the neck, $z^{*}=z_{\text {neck }}$. By $z_{\text {neck }}$ we mean here the point where $\rho(z)$ has a minimum. For the pear-like shape the neck does not exist and $z^{*}$ could be defined in a different way, see [6]. In the present work we are interested in the scission point configuration for which the neck is well defined. Then Eq. (1) turns into

$$
\frac{\delta}{\delta \rho}\left(E_{\mathrm{LD}}-\lambda_{1} V-\lambda_{2} R_{12}-\lambda_{3} \delta\right)=0 \quad \text { with } \quad \delta \equiv \frac{M_{L}-M_{R}}{M_{L}+M_{R}}=\frac{\pi}{V} \int \operatorname{Sign}\left(z-z^{*}\right) \rho^{2}(z) d z .
$$

The Euler-Lagrange equation for the variational problem (5) can be solved in the same way as Eq. (2). Some examples of the shapes at the critical point $R_{12}^{(c r i t)}$ for a few values of the mass asymmetry are shown in Fig. 3 a.
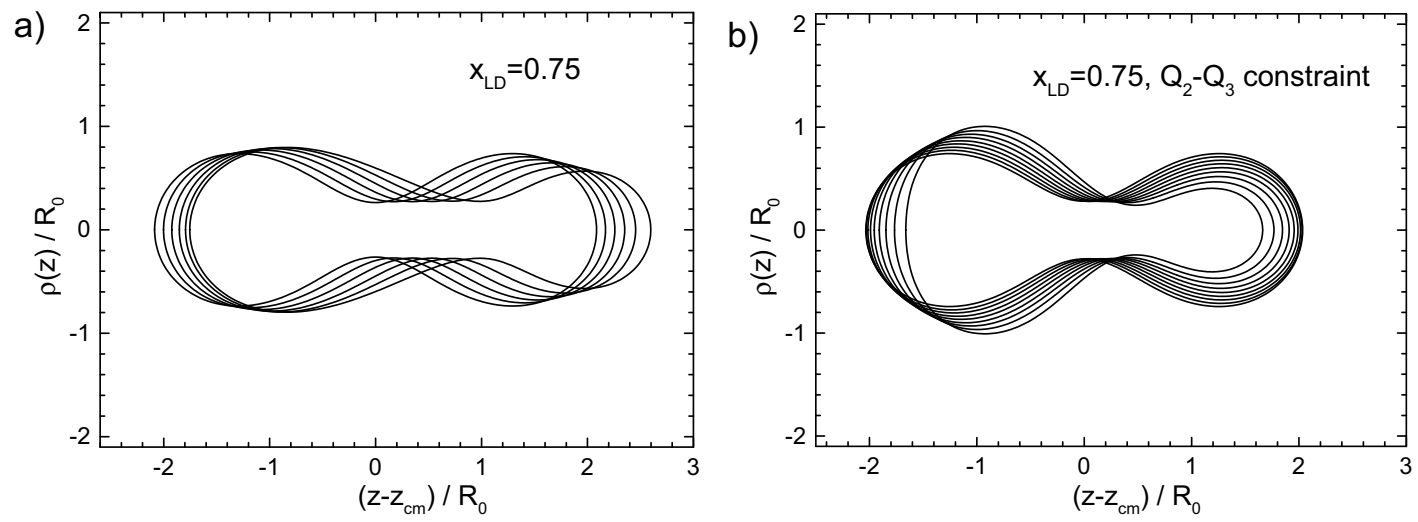

Figure 3. (a) The solutions of Eq. (5) at the maximal elongation $R_{12}^{(s c i .)}$ for a few values of the mass asymmetry $\delta=0,0.1, \ldots 0.5$; (b) The solutions of Eq. (7) at the maximal elongation $R_{12}^{(c r i t)}$ for few values of the mass asymmetry $\delta=0,0.1, \ldots 0.8$.

Besides $R_{12}$ and $\delta$ one could try another popular pair of constraints which are often used in the constrained Hartee-Fock calculations, namely, the quadrupole and octupole moments,

$$
Q_{2}=\frac{2}{V} \int d V r^{2} P_{2}(\cos \theta), \quad Q_{3}=\frac{1}{V} \int d V r^{3} P_{3}(\cos \theta) .
$$

The moments $Q_{2}$ and $Q_{3}$ can be defined independently of whether the neck exists or not. The use of quadrupole and octupole moments as constraints leads to the following variational problem

$$
\frac{\delta}{\delta \rho}\left(E_{\mathrm{LD}}-\lambda_{1} V-\lambda_{2} Q_{2}-\lambda_{3} Q_{3}\right)=0 .
$$

The Euler-Lagrange equation for the problem (7) can be solved in the same way as Eq. (2). The examples of solution of Eq. (7) for few value of the mass asymmetry are shown in the right part of Fig. 3.

Comparing the shapes at the scission point calculated by Eq. (5) and Eq. (7) for the same massasymmetry one can see that these two sets of shapes are rather different. The $Q_{2}, Q_{3}$ restrictions lead to scission shapes which are considerably "shorter" as compared with the scission shapes defined with $R_{12}, \delta$ restrictions. For an accurate description of the mass-asymmetric nuclear shape at the scission point it is necessary to construct an interpolation between the two sets of definitions for the elongation 
and mass asymmetry which are applied successfully at small deformations or for separated fragments. Such an interpolation can be achieved by the replacement of the $|z|$ and Sign z functions which appear in (1) and (5) by the smoothed absolute value and Sign functions

$$
|z| \Longrightarrow f_{2}(z) \equiv \sqrt{z^{2}+(\Delta z)^{2}}, \quad \operatorname{Sign} z \Longrightarrow f_{3}(z) \equiv \frac{z}{\sqrt{z^{2}+(\Delta z)^{2}}} .
$$

The expressions for the elongation and mass asymmetry change then into

$$
R_{12} \Longrightarrow \widetilde{R}_{12} \equiv \frac{\pi}{V} \int_{z_{1}}^{z_{2}} \sqrt{\left(z-z^{*}\right)^{2}+(\Delta z)^{2}} \rho^{2}(z) d z, \quad \delta \Longrightarrow \widetilde{\delta} \equiv \frac{\pi}{V} \int_{z_{1}}^{z_{2}} \frac{\left(z-z^{*}\right) \rho^{2}(z)}{\sqrt{\left(z-z^{*}\right)^{2}+(\Delta z)^{2}}} d z
$$

The replacement (8) contains an additional parameter - the smoothing width $\Delta z$. In principle, one can consider it as an additional collective parameter which has to be taken into account in the dynamical calculations. In the quasi-static limit one could expect that the value of $\Delta z$ is close to the neck radius in the scission region. In the calculations reported below $\Delta z$ was chosen as $\Delta z=0.25 R_{0}$. The calculated results do not change much if $\Delta z$ varies within the limits $0.1 R_{0} \lesssim \Delta z \lesssim 0.5 R_{0}$.
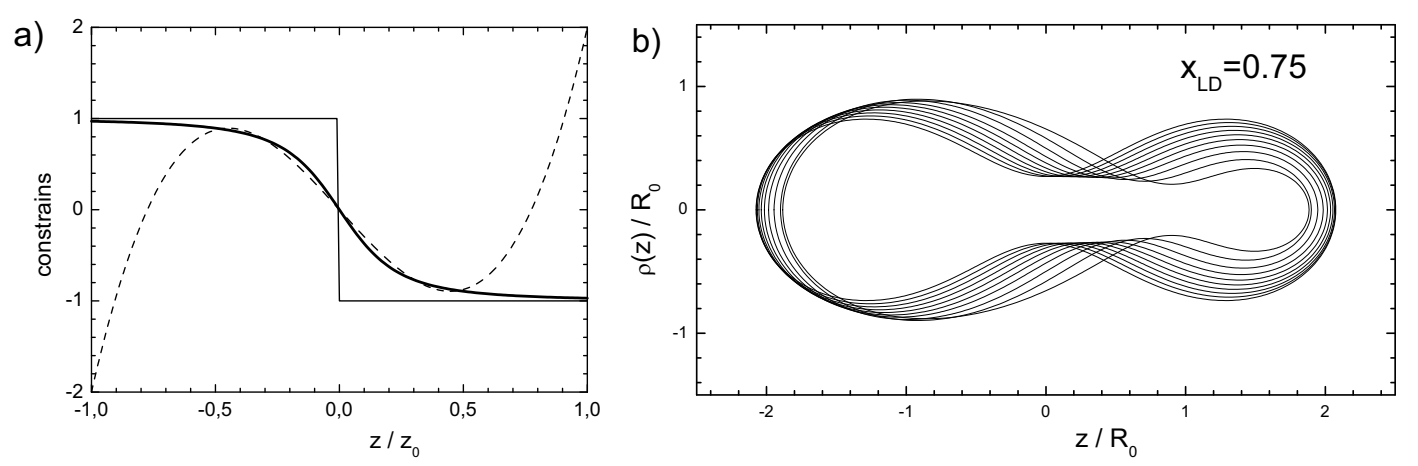

Figure 4. (a) Illustration of the constraints $\delta$ (solid), $P_{3}\left(z / z_{0}\right)$ (dash) and smoothed Sign-function (thick solid line); (b) The solutions of Eq. (10) $\left(\Delta z=0.25 R_{0}\right)$ at the maximal elongation $R_{12}^{(s c i .)}$ for few values of the mass asymmetry $\delta=0,0.1, \ldots 0.6$.

The use of the constraints given in (9) leads to the following equation

$$
\frac{\delta}{\delta \rho}\left[E_{\mathrm{LD}}-\lambda_{1} V-\lambda_{2} \widetilde{R}_{12}-\lambda_{3} \widetilde{\delta}\right]=0
$$

Eq.(10) can be solved in the same way as Eq.(2). Some examples of the shapes at the scission point $R_{12}^{(\text {crit) }}$ (maximal possible value of $\widetilde{R}_{12}$ at fixed $\lambda_{3}$ ) for a few values of the mass asymmetry $\widetilde{\delta}$ are shown in Fig. 4. The length of these shapes is in between those shown in Fig. 3. One notes that the neck radius at the scision point does not vary much with the mass asymmetry, $R_{\text {neck }} \approx 0.3 R_{0}$.

\section{The shell effects in the scission point configuration}

The optimal shapes given by the solutions of $(1,5,7,10)$ correspond to the minimum of the liquid drop energy. However, the shape of fissioning nuclei depends not only on the liquid drop part of the 
deformation energy but also on the shell effects. It was shown in [8] that due to the shell structure, in the deformation energy of actinide nuclei there exists a deep minimum corresponding to the shape at which one part is almost spherical due to the large shell correction of the double magic fragment ${ }^{132} \mathrm{Sn}$ and another part is very elongated. Clearly, such a shape can not be obtained within the variational approach (10) where the deformation of the fragments is completely fixed by the deformation dependence of the liquid-drop energy.

In order to account for the influence of shell effects on the shape of the scissioning nucleus, it is necessary to include into the optimal shapes procedure more degrees of freedom. For this purpose it was proposed in [7] to include in the variational procedure another two constraints which allow to vary the quadrupole moments of left and right fragments,

$$
\frac{\delta}{\delta \rho}\left[E_{\mathrm{LD}}-\lambda_{1} V-\lambda_{2} \widetilde{R}_{12}-\lambda_{3} \widetilde{\delta}-\lambda_{5} Q_{2 L}-\lambda_{6} Q_{2 R}\right]=0 .
$$

In (11) $Q_{2 L}$ and $Q_{2 R}$ are the quadrupole moments of left and right parts of nucleus while the limit between left and right is defined at the point $z_{\text {neck }}$ where the derivative $d \rho(z) / d z$ turns into zero. In what follows we are interested only in shapes at the scission point where the neck position is well defined. Solving (11) for large values of $\lambda_{5}$ and $\lambda_{6}$ one gets very elongated $\left(\lambda_{5}\right.$ and $\lambda_{6}$ both large positive) or very short shapes ( $\lambda_{5}$ and $\lambda_{6}$ both large negative) or shapes where one part is close to spherical and another very elongated (opposite sign of $\lambda_{5}$ and $\lambda_{6}$ ).
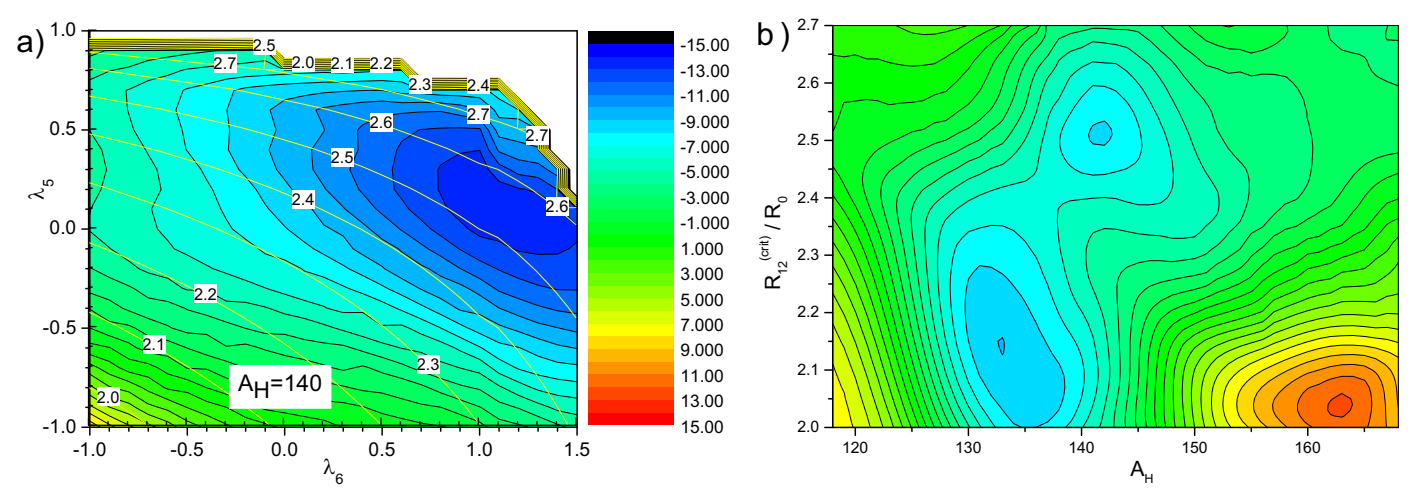

Figure 5. (color online) (a) The total deformation energy at the maximal elongation $R_{12}=R_{12}^{(\text {crit })}$ for the asymmetry corresponding to heavy fragment mass $A_{H}=140$ calculated with the solutions of Eq. (11) as function of Lagrange multipliers $\lambda_{5}$ and $\lambda_{6}$, see Eq. (11). The yellow contour lines show the value of $R_{12}^{(\text {crit })}$; (b) The shell component of the scission point deformation energy of ${ }^{236} \mathrm{U}$ as function of the heavy fragment mass number $A_{H}$ and the maximal elongation $R_{12}^{(\text {crit })}$.

For each value of the mass asymmetry the scission point turns in this case into the "scission surface". In order to visualize this scission surface we reduce it to a "scission line". At each point on the scission surface one can calculate the deformations of left and right fragments $Q_{L}$ and $Q_{R}$ and by the critical elongation $R_{12}^{(\text {crit })}$ (which is a function of $Q_{L}$ and $Q_{R}$ ). For each value of $R_{12}^{(c r i t)}$ the total deformation energy (liquid drop part plus shell correction) was minimized with respect to $\lambda_{5}$ and $\lambda_{6}$, i.e., one fixes the additional parameters $\lambda_{5}$ and $\lambda_{6}$ by the requirement that the total energy at critical elongation $R_{12}^{(\text {crit })}$ is minimal. In this way one gets the deformation energy at the scission point as function of the critical elongation $R_{12}^{(c r i t)}$. Figs. $5 \mathrm{~b}$ and 6 a show separately the shell correction and the liquid drop energy obtained as the result of such minimization. 
One clearly notices two minima in Fig. 5b. One, at short scission shape at $A_{H} \approx 134$ is caused by the large shell correction for ${ }^{132} \mathrm{Sn}$. The other minimum is caused by the "deformed shell" at $A_{H} \approx 142$. As will become clear below, these minima have a large influence on the fission fragment mass distribution and the total kinetic energy.

As one could expect, the liquid-drop energy grows with increasing mass asymmetry and decreases with growing critical deformation $R_{12}^{(\text {crit })}$ as seen on Fig. 6 a.
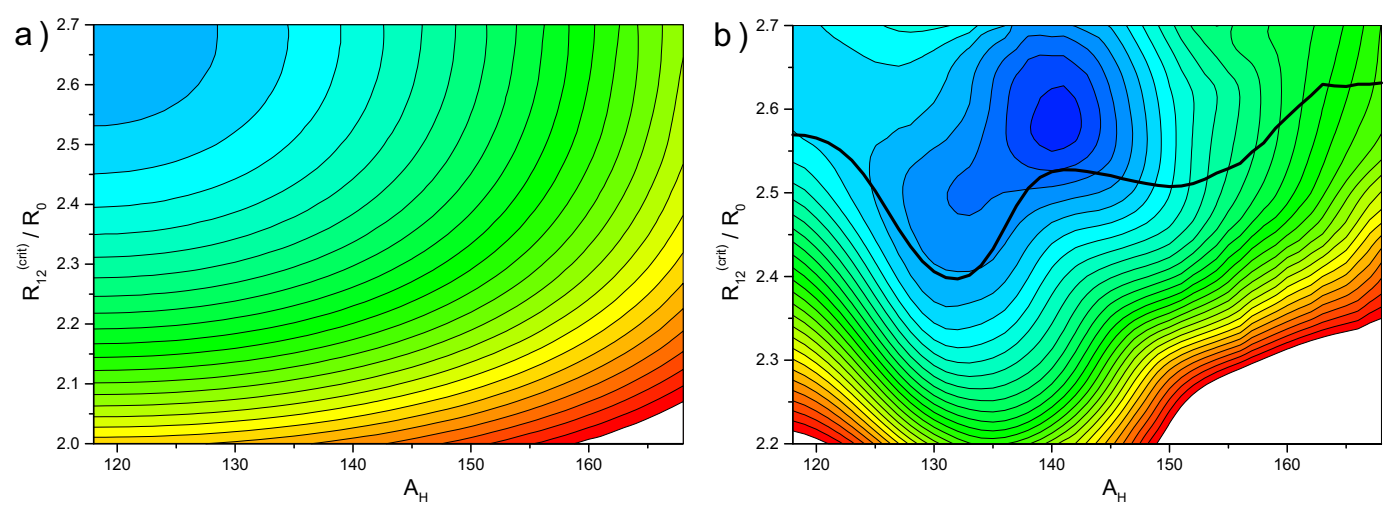

Figure 6. (color online) (a) The liquid drop component of the scission point deformation energy of ${ }^{236} \mathrm{U}$ as function of the heavy fragment mass number $A_{H}$ and the maximal elongation $R_{12}^{\text {(crit) }}$; (b) The total energy (liquid drop plus shell correction) for ${ }^{236} \mathrm{U}$ at the scission point as function of the heavy fragment mass number $A_{H}$ and the maximal elongation $R_{12}^{(\text {crit })}$. The thick line shows the mean value (14) of $R_{12}^{(\text {crit })}$.

The total scission-point deformation energy for ${ }^{236} \mathrm{U}$ is shown in Fig. 6b. Due to overlay of the liquid drop and shell components of the energy, the minimum around $A_{H}=134$ is no longer seen. However, it influences the calculated results for the fission observables (see below). The dependence of the fission fragments kinetic energy on the mass asymmetry is defined mainly by this value.

\section{The kinetic and excitation energy of fission fragments}

Keeping in mind that fission is a slow process, one could assume that during the fission process the state of the fissioning nucleus is close to statistical equilibrium, i.e. each point $q_{i}$ on the deformation energy surface is populated with a probability given by the canonical distribution,

$$
P\left(\delta_{i}, q_{i}\right)=e^{-\left(E\left(\delta_{i}, q_{i}\right)-Z\right) / T_{\text {coll }}}, \quad Z \equiv-T_{\text {coll }} \log \sum_{i} e^{-E\left(\delta_{i}, q_{i}\right) / T_{\text {coll }}} .
$$

Here $T_{\text {coll }}$ is a parameter characterizing the width of the distribution (12) in the space of deformation parameters. The energy $E\left(\delta_{i}, q_{i}\right)$ in (12) is the sum of the liquid-drop deformation energy (4) and of the shell correction $\delta E$, shown in Fig. 6b. The $\delta_{i}$ is the mass asymmetry of fissioning system and $q_{i}$ are the rest of collective parameters (elongation $R_{12}$, and Lagrange multipliers $\lambda_{5}$ and $\lambda_{6}$ ).

The distribution (12) is a basic assumption of the scission-point model suggested in [9] and developed further in [10-12], see also [13]. The parameters of this model were fitted in [9] to reproduce the numerous experimental data. The $T_{\text {coll }}$ was found to be close to $1 \mathrm{MeV}$. In the calculations shown below we use somewhat larger value $T_{\text {coll }}=1.5 \mathrm{MeV}$. 
The comparison of the calculated in [14] $T K E$ for few fission reaction with the available experimental results is shown in Fig. 7. In these calculations we define $T K E$ as the sum of the Coulomb interaction of spherical fragments immediately after scission and the prescission kinetic energy $K E_{\text {pre }}$,

$$
T K E=<E_{\text {Coul }}^{(\text {int })}(\delta)>+K E_{\text {pre }}, \text { with }<E_{\text {Coul }}^{(i n t)}(\delta)>\equiv \frac{1}{2} \sum_{i} \frac{Z_{L} Z_{H} e^{2}}{R_{12}^{(\text {crit })}\left(\delta, q_{i}\right)} P\left(\delta, q_{i}\right) / \sum_{i} P\left(\delta, q_{i}\right),
$$

where $e Z_{L}$ and $e Z_{H}$ are the charges of light and heavy fragments. The summation in the right part of (13) is carried out over $\lambda_{5 i}$ and $\lambda_{6 i}$. One can see from (13) that the Coulomb interaction energy depends crucially on the mean value of the distance between centers of mass of fragments

$$
<R_{12}^{(c r i t)}(\delta)>\equiv \sum_{i} R_{12}^{(c r i t)}\left(\delta, q_{i}\right) P\left(\delta, q_{i}\right) / \sum_{i} P\left(\delta, q_{i}\right)
$$

The small $<R_{12}^{(\text {crit })}(\delta)>$ leads to the large value of $<E_{\text {Coul }}^{(i n t)}(\delta)>$ and, on the contrary, the large $<R_{12}^{(\text {crit })}(\delta)>$ leads to the small value of $<E_{\text {Coul }}^{(\text {int })}(\delta)>$.

Like in [4] we define the $K E_{\text {pre }}$ from the energy balance, the sum of the prescission kinetic energy and the potential energy just before the scission should be the same as the sum of the ground state energy of mother nucleus and the neutron binding energy:

$$
E_{g s}\left(A_{L}+A_{H}\right)+B_{n}=E^{(j b s)}+K E_{p r e}
$$

i.e. we assume the "complete acceleration": the energy difference between the saddle and scission turns into the kinetic energy of relative motion of fragments, no dissipation takes place. By "just before scission" $E^{(j b s)}$ we mean here the mean value of the total deformation energy

$$
E^{(j b s)}(\delta) \equiv \sum_{i} E_{d e f}\left(\delta, \lambda_{5 i}, \lambda_{6 i}\right) P\left(\delta, \lambda_{5 i}, \lambda_{6 i}\right) / \sum_{i} P\left(\delta, \lambda_{5 i}, \lambda_{6 i}\right)
$$

The opposite extreme case would be the assumption of overdamped motion: all the energy difference between the saddle and scission turns into the heat, no prescission kinetic energy.

The comparison of the calculated and measured values of $T K E$ shown in Fig. 7 is in favor of "complete acceleration". Without contribution from $K E_{\text {pre }}$ the $T K E$ (dash curve in Fig. 7) would be too small.
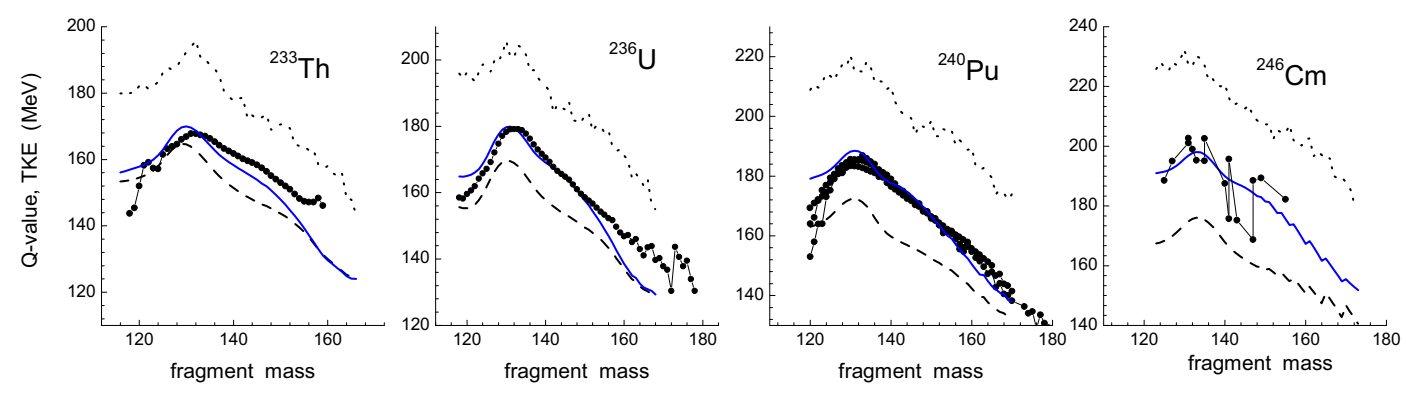

Figure 7. The total kinetic energy (13) calculated with (solid) and without (dash) account of fragments prescission kinetic energy. The experiments results (solid circles) are taken from [15-20]. The $Q$-values for fission of ${ }^{232} \mathrm{Th},{ }^{235} \mathrm{U},{ }^{239} \mathrm{Pu}$ and ${ }^{245} \mathrm{Cm}$ are shown by dotted lines. 
Note, that the calculated $T K E$ is rather close to experimental data. The position of maximum of $T K E$ and the drop at symmetric splitting are well reproduced. This can be considered as a confirmation that the potential energy just before scission and the mean value of $\left\langle R_{12}^{(c r i t)}\right\rangle$ are defined correctly.

The normalized mass distribution of the fission fragments $Y(\delta)$ can be expressed then in terms of the deformation energy at the critical deformation $R_{12}^{(\text {crit })}$,

$$
Y(\delta)=\sum_{i} P\left(\delta, R_{12}^{(c r i t)}, \lambda_{5 i}, \lambda_{6 i}\right)
$$

The summation in (17) is carried out over the Lagrange multipliers $\lambda_{5}, \lambda_{6}\left(R_{12}^{(\text {crit })}\right.$ is a function of $\left.\lambda_{5}, \lambda_{6}\right)$.

The calculated mass distribution (12)-(17) of the fission fragments for the reaction ${ }^{235} \mathrm{U}+n_{t h}$ is compared with the experimental data [21] in Fig. 8a. The calculated mass distribution is rather close to the experimental results. The presence and the position of two mass asymmetric peaks are well reproduced. At the symmetric splitting the calculated values are somewhat larger as compared with the experiment. The $T K E$ is one of two parts of the total energy release $Q, T K E+T X E=Q$. The
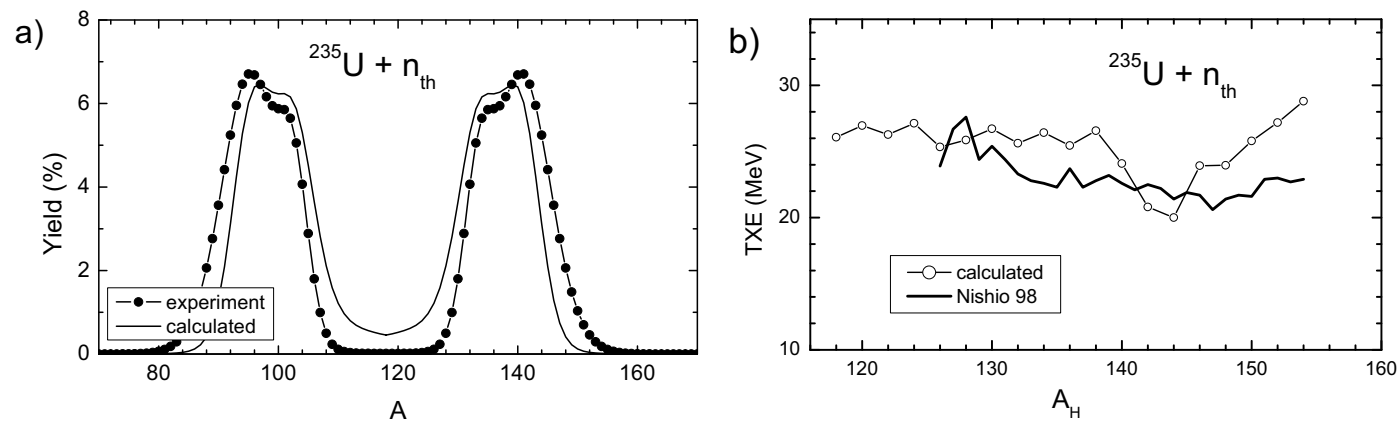

Figure 8. (a) The calculated $(12,17)$ and experimental $[21]$ values of the mass distribution of fission fragments in reaction ${ }^{235} \mathrm{U}+n_{t h}$; (b) The calculated (19) and experimental [22] values of the total excitation energy.

$Q$-value is the difference of the ground state energies

$$
Q \equiv E_{g s}\left(A_{L}+A_{H}\right)+B_{n}-E_{g s}\left(A_{L}\right)-E_{g s}\left(A_{H}\right)
$$

The $Q$-value does not depend on dynamics, it can be calculated within the macroscopicmicroscopic method or taken from the existing databases. Since the experimental value of $T K E$ is rather well reproduced by the present calculations, the calculated total excitation energy $T X E$

$$
T X E=Q-T K E
$$

is also quite accurate, see Fig. 8 b.

\section{The multiplicity of prompt neutrons}

After the neck rupture the fragments are de-excited by the emission of neutrons and $\gamma$-rays. When the excitation energy is smaller than neutron separation energy $S_{n}$ the $\gamma$-quanta are emitted. The energy 
available for $\gamma$-emission varies from $S_{n}$ to zero. So, on average the excitation energy available for neutron emission is given by $<T X E>-S_{n} / 2$. The average value of the total number $\bar{v}_{t o t}$ of prompt neutrons can be estimated by the relation

$$
\bar{v}_{t o t} \approx<T X E>/ \bar{S}_{n}-1 / 2, \text { with } \bar{S}_{n}=5.7 \mathrm{MeV} .
$$

In order to calculate the dependence of $\bar{v}$ on the fragments mass number $A$ one needs to know how the excitation energy is shared between the fragments. Here we split the last stage of the fission into two steps. On first step the rapid neck rupture takes place. The nucleus turns into two fragments with the same mass asymmetry and the same distance between centers of mass as just before scission. The shape of the fragments immediately after scission is assumed to correspond to the minimum of the liquid drop energy. It was shown in [7] that the optimal shape of the fragments placed at the distance $R_{12}^{(c r i t)}$ is very close to two spheres. The contribution to energy from quadrupole and higher order deformation can be neglected. So, in present work we assume that immediately after scission the two spherical fragments are formed. The energy immediately after scission consists of the energy of light and heavy spherical fragments plus the energy of their Coulomb interaction,

$$
E^{(i a s)}(A)=E^{(s p h)}\left(A_{L}\right)+E^{(s p h)}\left(A_{H}\right)+E_{\text {Coul }}^{(i n t)}\left(R_{12}, \delta\right) .
$$

The primary excitation energy $E^{(i a s)}-E^{(j b s)}$ is shared between the fragments. In the recent work [23] it was shown that difference of the excitation energy of light and heavy fragment is not so large, only few $\mathrm{MeV}$. So, for simplicity we will assume here that the primary excitation energy of light and heavy fragments due to the neck rupture is the same. This excitation energy for ${ }^{236} \mathrm{U}$ is shown in Fig. 9b. On
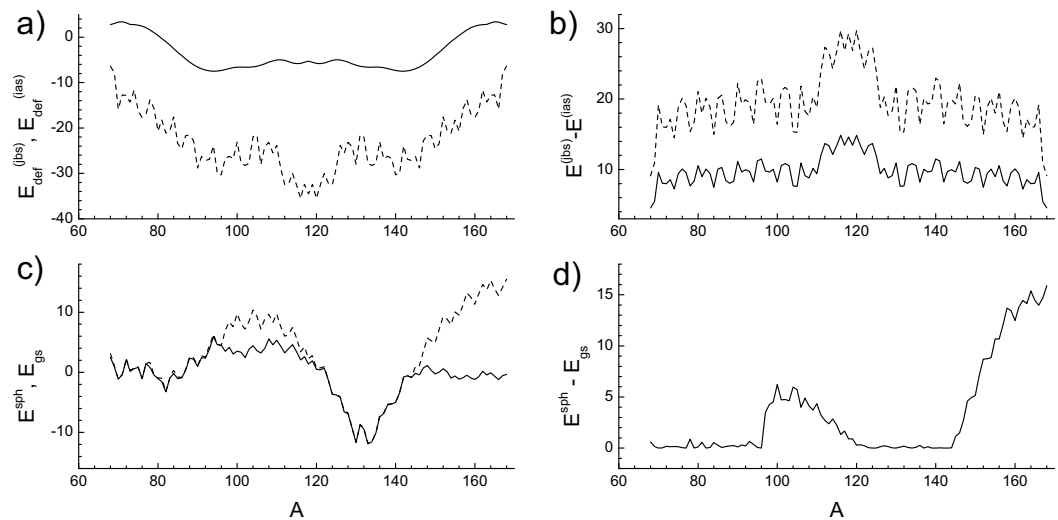

Figure 9. (a) The deformation energy of ${ }^{236} \mathrm{U}$ just before scission (solid) and immediately after scission (dash) as function of fragments mass number $A$; (b) the primary excitation energy $E_{d e f}^{j b s}-E_{d e f}^{i a s}$; (c) the deformation energy $E^{(s p h)}(A)$ at spherical shape (dash) and $E_{g s}(A)$ at the ground state (solid); (d) the extra deformation energy (22) of fission fragments; e) the excitation energy (23) of fission fragment. All energies in this figure are given in $\mathrm{MeV}$.

the second stage of fission process the fragments relax to the ground state shape and gain some extra deformation energy

$$
\Delta E_{\text {def }}(A)=E^{(s p h)}(A)-E_{g s}(A)
$$

So, the total excitation energy of one fragment is given by the sum

$$
E_{x}(A)=\left[E^{(j b s)}(A)-E^{(i a s)}(A)\right] / 2+\Delta E_{d e f}(A) .
$$


In Fig. 10 we compare the excitation energy available for the prompt neutron emission, $E_{x}(A)-0.5 S_{n}$ with the experimental value of neutron multiplicity $[22,24]$ multiplied by the half of two-neutron separation energy $S_{2 n}$ (in order to remove the rapid fluctuations due to the odd-even effect in $S_{n}$ ). One can see that there is some discrepancy up to $5 \mathrm{MeV}$ at large mass asymmetries, but on the average the saw-tooth structure is rather well reproduced.

Having at one's disposal the excitation energy $E_{x}(A)$ one can calculate the total number of prompt neutrons

$$
\bar{v}_{t o t}=\sum_{A}\left[E_{x}(A) / S_{n}(A)-1 / 2\right] Y(A) .
$$

The comparison of calculated $\bar{v}_{t o t}$ with the available experimental results is shown in Fig. 10b. On

a)

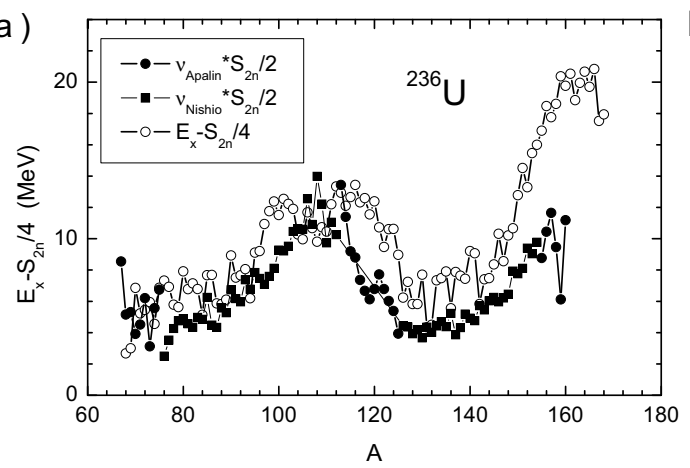

b)

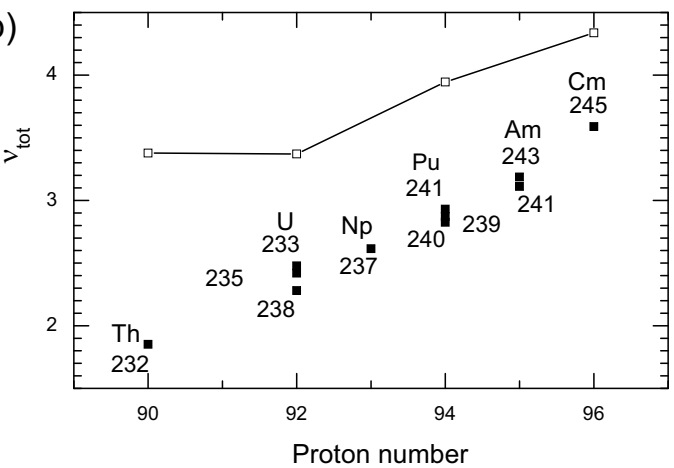

Figure 10. (a) The excitation energy (23) available for prompt neutron emission (open circles) and the experimental results for neutron multiplicity $[22,24]$ multiplied by half of two-neutron separation energy; (b) The calculated value of total neutron multiplicity (24) (open squares) and the experimental results [25] (solid squares).

average the dependence of $\bar{v}_{t o t}$ on the proton number of fissioning nuclei is qualitatively reproduced. The calculated value of $\bar{v}_{t o t}$ are however larger than experimental by (0.5-0.9). The source of this discrepancy can be related to the use of very simple estimate (20) for the neutron multiplicity and use of the quasistatic approximation (12) for the mass distribution.

For more accurate description of mass distribution the dynamical approach to the fission process seems necessary.

\section{Summary}

The calculations presented in this work show that the optimal shape prescription offers an accurate definition of the shape of fissioning nuclei just before the scission (the scission point configuration). Within the quasi-static picture the information on the shape and energy at the scission point can be used for the evaluation of the quantities, measured in fission experiments like the mass distribution, the kinetic and excitation energy of fission fragments, the multiplicity of prompt neutrons.

The results of calculations show that the calculated mass distribution and the excitation energy for the reaction ${ }^{235} \mathrm{U}+n_{t h}$ are very close to the experimental values. The calculated distributions of the total kinetic energy of fission fragments for the fission of ${ }^{232} \mathrm{Th},{ }^{235} \mathrm{U},{ }^{239} \mathrm{Pu}$ and ${ }^{245} \mathrm{Cm}$ by thermal neutrons are also in rather good agreement with the experimental data.

The sawtooth structure of the neutron multiplicity is qualitatively reproduced. The dependence of total multiplicity of prompt neutrons on the proton number of fissioning nucleus is reproduced only qualitatively. The calculated value of $\bar{v}_{t o t}$ are larger than experimental by $(0.5-0.9)$. 
The reason for the discrepancy between calculated and experimental results for average total prompt neutron multiplicities may be too crude approximation of Eqs. $(20,24)$ for the relation between the excitation energy and neutron multiplicity. There could be also other reasons, like the use of assumption that each point on the deformation energy surface is populated with a probability given by the canonical distribution (12).

\section{Acknowledgements}

The author would like to express his gratitude to the Research Laboratory for Nuclear Reactors, Tokyo Institute of Technology, for the hospitality during his visits to Japan. The contribution includes partly the results of "Comprehensive study of delayed-neutron yields for accurate evaluation of kinetics of high-burn up reactors" entrusted to the Tokyo Institute of Technology by the Ministry of Education, Culture, Sports, Science and Technology of Japan (MEXT).

\section{References}

[1] V. M. Strutinsky, Nucl. Phys. 3, 449 (1966); Nucl. Phys. A95, 420 (1967); Nucl. Phys. A122, 1 (1968).

[2] M. Brack, J. Damgaard, A.S. Jensen et al, Rev. Mod. Phys. 44, 320 (1972).

[3] V.M. Strutinsky, N.Ya. Lyashchenko, N.A. Popov, Nucl. Phys.46, 639 (1963).

[4] F.A. Ivanyuk, Phys. Scr. 89, 054012 (2014).

[5] N. Bohr and J. A. Wheeler, Phys. Rev. 56 (1939) 426.

[6] F.Ivanyuk, Int. J. Mod. Phys. E 18, 130 (2009).

[7] F.A. Ivanyuk, Physics Procedia 47, 17 (2013).

[8] V.V. Pashkevich, Nucl. Phys. A 169, 275 (1971).

[9] B.D. Wilkins, E.P. Steinberg, and R.R. Chasman, Phys. Rev. C 14, 1832 (1976).

[10] J. Moreau, K. Heyde, and M. Waroquier, Phys. Rev. C 28, 1640 (1983).

[11] A. Ruben, H. Marten, D. Seeliger, Zeit .für Physik A Hadrons and Nuclei 338, 67 (1991).

[12] S. Panebianco, J.-L. Sida, H. Goutte et al, Phys. Rev. C 86, 064601 (2012).

[13] H.-J. Krappe and K. Pomorski, Theory of Nuclear Fission, Springer Verlag, Heidelberg, 2012.

[14] F.A. Ivanyuk, S. Chiba and Y. Aritomo, Phys. Rev. C 90, 054607 (2014).

[15] A.I. Sergachev, V.G. Vorob'eva, B.D. Kuz'minov et al, Yad. Fiz. 7, 778 (1968).

[16] F.-J. Hambsch, H.-H Knitter, C. Budtz-Jorgensen and J. Theobald, Nucl. Phys. A 491, 56 (1989).

[17] C. Tsuchiya, Y. Nakagome, H. Yamana et al, J. Nucl. Sc. Techn. 37, 941 (2000).

[18] C. Wagemans, E. Allaert, A. Deruytter et al, Nucl. Phys. A 380, 61 (1982).

[19] K. Nishio, Y. Nakagome, I. Kanno, I. Kimura, J. Nucl. Sc. Techn. 32, 404 (1995).

[20] A. Ramaswami, S. Prakash, S. B. Manohar et al, Phys. Rev. C 16, 716 (1977).

[21] S. Zeynalov, V. Furman, F.-J. Hambsch et al, in Proceedings of the 13th International Seminar on Interaction of Neutrons with Nuclei (ISINN-13), Dubna, Russia, May 25-28, 2005 (Joint Institute for Nuclear Research, Dubna, 2006), p. 351.

[22] K. Nishio, Y. Nakagome, H. Yamamoto, I. Kimura, Nucl. Phys. A 632, 540 (1998).

[23] N. Carjan, F.-J. Hambsch, F.A. Ivanyuk, P. Talou, Physics Procedia 59, 173 (2014).

[24] V.F. Apalin et al., Nucl. Phys. 71, 553 (1965).

[25] T. Ohsawa, J. Nucl. Radiochem. Sci. 9, 19 (2008). 